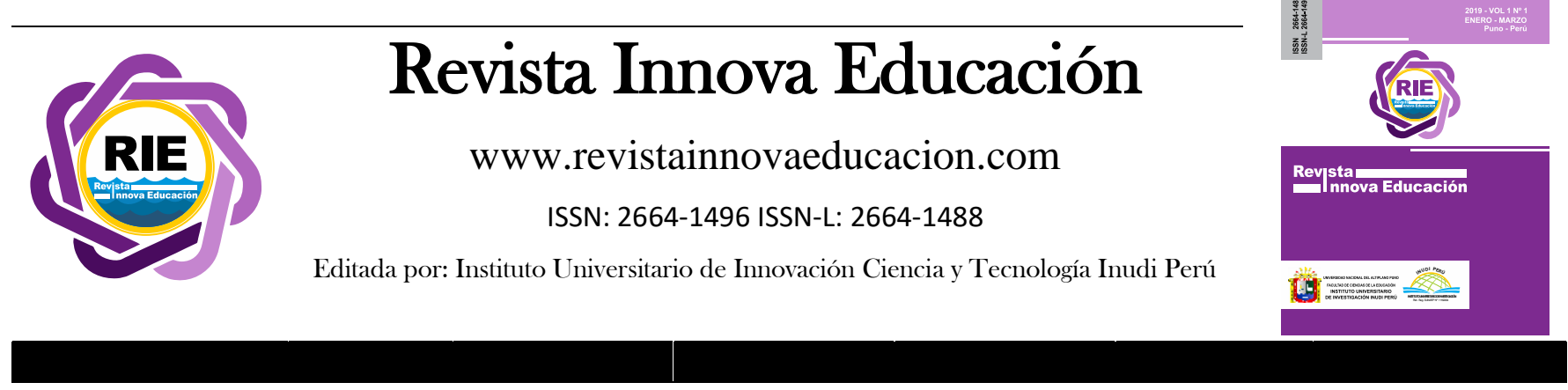

\title{
Análisis crítico del discurso como metodología de comprensión de las ideologías
}

\author{
Critical analysis of discourse as a methodology for understanding ideologies
}

\author{
Javier Núñez
}

DOI: https://doi.org/10.35622/j.rie.2019.04.007

Universidad Nacional del Altiplano, Perú.

Recibido el 22/07/2019/ Aceptado el 19/10/2019/

ARTíCULO ORIGINAL
PALABRAS CLAVE
Discurso,
ideología,
interpretación,
literacidad crítica,
lectura

\section{KEYWORDS}

Discourse, critic, critical literacy, comprehension, ideology, reading
El objetivo fue evaluar el impacto de la enseñanza del análisis crítico del discurso como metodología de comprensión de las ideologías en los estudiantes de Sociología de la Universidad Nacional del Altiplano Puno, 2018. Este tema se enmarca en el marco de la literacidad crítica; la cual hace referencia al desarrollo de la competencia para leer adecuadamente las ideologías de los discursos. Los grupos "A" (grupo control) y "B" (grupo experimental) del segundo semestre conformaron la población de estudio. Para el recojo de los datos se ha utilizado la rúbrica. El objetivo de la investigación se alcanzó mediante el uso de la técnica estadística Diferencia en diferencias. Los resultados sugieren que los estudiantes del grupo experimental mejoraron el nivel de comprensión de las ideologías en un promedio de 5 puntos a diferencia de los estudiantes del grupo control. El cual es significativo en términos estadísticos a un nivel de $99 \%$ de confianza. Por lo tanto, se concluye que la enseñanza del análisis crítico del discurso y la literacidad crítica tiene un impacto positivo en la comprensión de las ideologías.
The objective was to evaluate the impact of teaching critical discourse analysis as a methodology for understanding ideologies on students of Sociology at the Universidad Nacional del Altiplano Puno, 2018. This topic is framed within the framework of critical literacy, which refers to the development of competence to read adequately the ideologies of discourses. Groups "A" (control group) and "B" (experimental group) of the second semester formed the study population. To collect the data The rubric was used. The research objective was achieved through the use of the statistical technique Difference in differences. The results suggest that the students of the experimental group improved the level of understanding of ideologies by an average of 5 points as opposed to the students of the control group. This is statistically significant at a level of $99 \%$ confidence. Therefore, it is concluded that teaching critical discourse analysis and critical literacy has a positive impact on the understanding of ideologies. 


\section{INTRODUCCIÓN}

Conocemos el mundo tentativamente, a medida que lo designamos con palabras y lo construimos sintácticamente en enunciados, es decir, el mundo adquiere sentido solo en la medida en que lo traducimos lingüísticamente (Chillón, 1998). Mediando el lenguaje construimos la realidad (o al menos construimos una representación de ella), y nos construimos como integrantes de un grupo social y como personas humanas. En ese sentido, para conocer la cultura, la filosofía, la cosmovisión, la ideología de un pueblo, o grupo social, necesitamos estudiar sus discursos.

El análisis crítico del discurso, en la actualidad, es un campo de investigación importante en el estudio de las ideologías. Son muchos los trabajos que se realizan en el mundo. Se dice que no hay textos neutros, sin ninguna ideología; más bien los textos reproducen y legitiman alguna ideología. Es por eso que hoy en día se da énfasis a la comprensión de las ideologías a través de la lectura crítica. En tal sentido, este trabajo es importante porque la educación superior tiene la obligación de formar lectores críticos y multidisciplinarios, para que los estudiantes logren el desarrollo de la competencia de lectura como experiencia de interpretación crítica, de manera que puedan evaluar, construir juicios, tomar posturas y decidir (Serrano de Moreno, 2008). Asimismo, el presente trabajo se realizó para proporcionar a los estudiantes y docentes una guía de lectura de las ideologías sobre la base del análisis crítico del discurso y la literacidad critica.

La lectura crítica fomenta el desempeño social y político del individuo al interior de su comunidad, orientando la proyección del proceso lector hacia la esfera de los asuntos públicos y de participación ciudadana activa y consciente (Cardona y Londoño, 2017). Por lo tanto, es importante adquirir habilidades de lectura crítica, porque ayuda a mejorar los proyectos académicos como la redacción y evaluación de trabajos de investigación y artículos científicos (García, 2014). Para fortalecer la alfabetización académica y la lectura crítica en los estudiantes es necesaria mayor inter y transdisciplinariedad (Benavides y Sierra, 2013). En ese sentido, la escuela debe formar sujetos críticos que entiendan sus mundos y participen en los procesos de transformación de sus realidades, ya que el contexto socio cultural es susceptible de ser transformado desde la lectura crítica (Rocha, 2016).

Ochoa y Moreno (2013) — en su estudio sobre la lectura crítica realizado en estudiantes de Licenciatura en Español y Filología Clásica de la Universidad Nacional de Colombia y de Español de la Pontificia Universidad Javeriana - concluyen que los estudiantes lograron identificar la intención del autor, la tesis que quería defender, y algunos mecanismos que emplea el autor para lograr su propósito comunicativo; no obstante, se observó dificultades al asumir una posición crítica frente al contenido y forma del texto. Por otro lado, Peña (2011) concluye que los estudiantes que ingresan a la universidad no tienen los conocimientos previos requeridos para la lectura crítica y la escritura académica; por lo tanto es necesario contribuir a la formación de los estudiantes para leer de manera crítica, reflexiva, analítica. En ese sentido, Maya (2011) demostró en su estudio que los cursos relacionados con las prácticas de la lectura y la escritura orientados desde la Lingüística Textual y el Análisis Crítico del Discurso presentan mejores resultados en análisis crítico y producción textual que aquellos orientados bajo teorías tradicionales.

Arias (2018) señala, en su estudio, que la lectura crítica permite desarrollar el pensamiento, ya que ayuda a analizar el lenguaje desde distintas perspectivas y la forma de entender el mundo. La misma autora agrega que la lectura crítica ayuda a comprender e identificar la realidad histórico-social en forma contextualizada. De manera que, como señala Martínez (1995), existe la necesidad de enfrentar críticamente los discursos periodísticos, por ejemplo la televisión, 
diseñando estrategias que formen espectadores responsables, críticos y participativos de los medios de comunicación. En ese sentido, la actividad pedagógica de enfoque crítico debe centrarse en orientar al lector a examinar los contenidos de lectura desde una perspectiva de diálogo abierto (Abouchaar, 1997). Asimismo, existe la necesidad de análisis y reflexión sobre lo que se mira y ve, se oye, escucha e interactúa (Fainholc, 2006).

Zárate (2019) concluye que las habilidades lectoras que se sustentan en la literacidad crítica ayudan a cuestionar el texto en vez de asumirlo pasivamente; a desarrollar interpretaciones alternativas y contextualizadas, y a diferenciar su opinión personal de la del autor. La lectura transversal puede ser una herramienta para formar lectores críticos en la esfera pública (Aguilar, 2008). Este autor explica que la lectura transversal es el conjunto de experiencias culturales que poseen las personas y que en un momento determinado les sirve para comprender e interpretar la realidad. Por su parte, Silva (2002) afirma que el desarrollo de una lectura crítica resulta de pensar, leer, escribir y debatir de un modo que vaya más allá de la mera opinión. Finalmente, las variables como la lectura crítica, la lectura pragmática, la lectura semántica y la lectura del mundo se han originado de los estudios sobre la lectura de enfoque sociocultural (Méndez, Arbeláez, Serna, Espinal y Gómez, 2014).

Alvarado (2012) concluye que las lecturas críticas identifican las estrategias comunicacionales que los medios masivos muestran a la audiencia en mensajes precodificados (efectos) y reconocen que detrás del mensaje percibido existen determinadas posturas ideológicas. Por otro lado, Romano y López (2016) concluyen que la comprensión del discurso mediático exige una formación crítica en alfabetización mediática. Asimismo, Barrio y Pérez (1997) observan, en el taller que realizaron, las actitudes de crítica reflexiva frente al fenómeno publicitario, es decir, los alumnos reconocían con facilidad la «publicidad engañosa» de productos cuyos mensajes transmitían usos que no se ajustaban a la realidad. Por su parte, Díaz, Bar y Ortiz (2015) advierten ciertas habilidades de lectura crítica en el análisis textual y en la interpretación global, en la identificación de las intenciones del autor y el reconocimiento de fuentes de autoridad.

Avendaño de Barón (2016) identifica que la mayoría de los docentes concibe la lectura crítica de modo parcial y desenfocado, ya que se concentran solo en prácticas interpretativas y argumentativas, y no en procesos reflexivos, evaluativos y críticos. Castro y Martínez (2017) sugieren en su estudio que los docentes reconocen la importancia de la lectura crítica, pero aún presentan ciertas dificultades para potenciar este nivel de comprensión lectora en sus estudiantes de ELE, ya que predomina el desarrollo de actividades de comprensión de lectura en un nivel literal. Soria (2015) determina - en el diagnóstico de las estrategias metodológicas utilizadas en mejoramiento de la lectura crítica del centro de Educación Básica Enrique Rodríguez Fabregatque más del $60 \%$ de los docentes no utilizan estrategias metodológicas para el mejoramiento de la lectura crítica, y el 74\% no aplican motivación a su clase de lectura, ya que utilizan la metodología tradicionalista.

\section{ANÁLISIS CRÍTICO DEL DISCURSO}

\subsection{Generalidades}

El análisis crítico del discurso (ACD) tiene como objetivo desnaturalizar las ideologías mediante el estudio de las prácticas discursivas que tienen una aparente objetividad y neutralidad; ya que el lenguaje es un arma que, aparte de reproducir relaciones de poder, refleja formas de 
resistencia a ideologías dominantes (Vich y Zavala, 2004); es decir, uno de los objetivos del ACD es desmitificar los discursos mediante el descifrado de las ideologías (Wodak, 2003). En el ACD se consideran tres dimensiones de análisis (Van Dijk, 2016; Van Dijk, 1994): a) Discurso (uso del lenguaje); b) cognición (comunicación de creencias), y c) sociedad (interacción social). Entonces, en el estudio del discurso se describen tres dimensiones integradas: a) ¿cómo influye el uso del lenguaje en las creencias y en la interacción, o viceversa?, b) ¿cómo influyen algunos aspectos de la interacción en la manera de hablar?, y c) ¿cómo controlan las creencias el uso del lenguaje y la interacción? (Van Dijk, 2008a).

\subsection{El papel del discurso}

Guzmán (2016) afirma que el discurso — desde la perspectiva del análisis crítico — tiene que ver con la "reproducción discursiva del poder y la dominación" y sobre todo con el acceso al "discurso y los eventos comunicativos". Por su parte, Van Dijk (2009) plantea las siguientes preguntas para identificar a los grupos que controlan el discurso (ejercen el poder) y los que tienen un acceso restringido (dominados): ¿quién puede hablar o escribir a quién, sobre qué, cuándo y en qué contexto? O bien, ¿quiénes pueden participar en tales eventos comunicativos cumpliendo los diversos papeles del receptor? En suma, el discurso es una práctica social, un instrumento para crear la vida social; es decir, una forma de acción entre las personas que se construye mediante el uso lingüístico contextualizado (oral o escrito); es una parte de la vida social (Calsamiglia y Tusón, 1999).

\subsection{El poder social y el control de la mente}

De hecho, los grupos que controlan los discursos tienen más posibilidades de controlar las mentes y las acciones de los otros; de modo que es necesario conocer cómo las mentes controlan nuestras acciones y cómo, a partir de ello, se puede influenciar la mentalidad de la gente, a través del texto o el habla (Van Dijk, 1999). El mismo autor agrega que el control de la mente tiene que ver con la adquisición de creencias sobre el mundo a través del discurso y de la comunicación.

Enseguida se mencionan cuatro puntos que sugieren que el control discursivo de la mente es una forma de poder y de dominio (Van Dijk, 1999):

a) Los receptores u oyentes tienden a aceptar las creencias (conocimientos y opiniones) transmitidas por el discurso de las fuentes que consideran autorizadas, fidedignas o creíbles.

b) En otras ocasiones, los participantes están obligados a ser receptores del discurso (por ejemplo, en la educación y/o actividades laborales.

c) A veces no existen otros discursos alternativos sobre el mismo tema.

d) Sucede, también, en algunas ocasiones que los receptores no poseen conocimientos y creencias necesarias para desafiar los discursos o la información a que están expuestos (Wodak, 1987). 


\section{LITERACIDAD CRÍTICA}

La literacidad crítica está relacionada con la gestión de la ideología de los discursos (al leer y escribir). En ese sentido, la literacidad engloba conocimientos, habilidades y actitudes y valores derivados del uso generalizado, histórico, individual y social del código escrito (Cassany, 2005). Según este autor, la literacidad crítica tiene los siguientes elementos: Código escrito (conocimiento de las unidades léxicas del idioma, de las reglas gramaticales y de las normas de uso de las mismas); géneros discursivos (conocimiento y dominio de los géneros discursivos particulares); roles de autor/lector (conocimiento y uso de los roles que adoptan el autor y el lector en cada uno de los géneros mencionados); organización social (conocimiento y participación en las instituciones en que se desarrollan las prácticas discursivas); valores y representaciones (prácticas discursivas que desarrollan valores y concepciones sobre el mundo); formas de pensamiento (desarrollo de pensamiento propio, vinculado con la escritura, como la búsqueda de objetividad, el razonamiento científico o la capacidad de planificación del discurso).

\subsection{Leer críticamente}

Ser crítico al leer y escribir significa ser capaz de gestionar la ideología de los escritos, es decir, ser crítico significa realizar las siguientes operaciones (Cassany, 2005):

- Situar el texto en el contexto sociocultural de partida: Hay que realizar las siguientes operaciones: Identificar el propósito y situarlo en el entramado social de propósitos e intereses, reconocer el contenido incluido o excluido en el discurso, identificar las voces incorporadas o silenciadas de otros autores, citas, referencias, Caracterizar la voz del autor.

- Reconocer y participar en la práctica discursiva. Se debe realizar las siguientes operaciones: Saber interpretar el escrito según su género discursivo; reconocer las características socioculturales propias del género; conocer las prácticas lectoras y escritoras que se desarrollan con este género.

- Calcular los efectos que causa un discurso en la comunidad: Se debe llevar a cabo las siguientes operaciones: Tomar conciencia de la propia situacionalidad del lector; calcular las interpretaciones de los otros, sobre todo de los miembros más próximos de nuestra comunidad o de las autoridades; integrar estas interpretaciones en un valor o impacto global, que suma diferentes interpretaciones de un escrito.

\section{IDEOLOGÍA}

Desde la perspectiva de Van Dijk (2008b), la ideología tiene que ver con un sistema de puntos de vista e ideas sociales; es decir, es un sistema cognoscitivo de prohibiciones y creencias, que determina la identidad social de un grupo. En el ACD, la ideología permite el establecimiento y la conservación de relaciones desiguales de poder (Wodak, 2003). En ese sentido, las ideologías se utilizan como fundamento de dominación y resistencia; es decir, se usan para explicar el "mundo en general" (por ejemplo, la religión) y el "mundo social y económico" (por ejemplo, el capitalismo); además, tienen una dimensión normativa ("resumen de lo que los miembros del 
grupo deben hacer o no deben hacer"; por ejemplo, luchar contra la opresión) (Van Dijk, 2006). Las ideologías tienen que ver con la representación de la estructura mental de los objetivos e intereses fundamentales de un grupo: sociales, económicos y/o culturales (Van Dijk, 2003). En suma, la ideología es el punto de vista, el sesgo, la mirada o la subjetividad inevitable que esconde cualquier texto; es decir, todo discurso no necesariamente refleja la realidad, sino que el discurso tiene un autor detrás (individual o colectivo) que forzosamente vive en algún lugar del mundo y en una época, por lo que necesariamente su visión de la realidad es parcial, sesgada, personal (Cassany, 2005).

\subsection{Características de las ideologías}

Van Dijk (2008b) señala las siguientes características: a) Las ideologías son cognitivas (son ideas, pensamientos, creencias sociales, juicios y valores); b) las ideologías son sociales (tanto los grupos dominantes como los dominados tienen ideologías que controlan su propia identificación, objetivos y acciones); c) las ideologías son sociocognitivas (en los sistemas sociales, las creencias actúan como una interfaz entre lo cognitivo y lo social); d) Las ideologías no son verdaderas ni falsas (ya que tienen que ver con la forma como un grupo social ha armado su sistema de creencias); e) un grupo puede tener ideologías vagas o sólidas (hay personas que tienen aparentemente ideologías contradictorias); f) proporcionan coherencia (ideológica) a las creencias de un grupo y especifican qué valores son importantes para el grupo.

\section{MATERIALES Y MÉTODOS}

\subsection{Población y muestra}

La población de estudio estuvo conformada por la totalidad de los estudiantes de segundo semestre de la Escuela Profesional de Sociología de la Universidad Nacional del Altiplano de Puno, que cursan el semestre académico 2018 II.

Tabla 1 Población de estudio

\begin{tabular}{lccc}
\hline \multirow{2}{*}{ Grupos } & \multicolumn{3}{c}{ Estudiantes } \\
\cline { 2 - 4 } & Varones & Mujeres & Total \\
\hline "A" & 14 & 13 & 27 \\
"B" & 12 & 11 & 23 \\
\hline Total & 26 & 24 & 50 \\
\hline
\end{tabular}

FUENTE: Oficina de coordinación académica de la Facultad de Ciencias Sociales (UNA-Puno ${ }^{1}$ )

Como la población es de tamaño reducida, no se trabajó con ninguna muestra; sino con toda la población en su totalidad. Se seleccionó el grupo "B” como grupo experimental; y el grupo "A" como grupo control. En el grupo experimental se aplicó la metodología del análisis crítico del discurso y la literacidad crítica.

${ }^{1}$ Sigla: Universidad Nacional del Altiplano de Puno 


\subsection{Técnicas e instrumentos de recolección de datos}

La técnica que se usó para esta investigación fue la rúbrica. Como instrumento se usó la ficha de rúbrica.

\subsection{Tratamiento estadístico de datos}

Para alcanzar el objetivo de la investigación se utilizó la técnica econométrica de Diferencia en Diferencias (DID, por sus siglas en inglés). Con este método se calculó las diferencias en la evolución temporal de cada grupo (primera diferencia) para posteriormente ver la diferencia en el cambio que se produjo entre ambos grupos (segunda diferencia). Esta técnica consiste en comparar los cambios en el tiempo (entre preprueba y posprueba) en la variable dependiente entre el grupo experimental y el grupo control.

Esta técnica se estima a través de la siguiente ecuación de regresión:

$$
Y_{i t}=\beta_{0}+\beta_{1} D_{i}+\beta_{2} P_{t}+\alpha\left(D_{i} \times P\right)_{t}+\varepsilon_{i t}
$$

Donde:

$\mathrm{Y}_{\mathrm{it}} \quad=$ variable de interés en el periodo $\mathrm{t}$, en el individuo $\mathrm{i}$.

$D_{i} \quad=\left\{\begin{array}{l}1 \text { (si el individuo es del grupo experimental }) \\ 0(\text { si el individuo es del grupo control })\end{array}\right.$

$\mathrm{P}_{\mathrm{t}} \quad=\left\{\begin{array}{l}1 \text { (si el dato es de prueba de salida }) \\ 0(\text { si el dato es de prueba de entrada })\end{array}\right.$

$\varepsilon_{\mathrm{it}} \quad=$ término error que tiene media cero y varianza $\sigma^{2}$.

$\beta_{0}, \beta_{1}, \beta_{2}, \alpha \quad=$ parámetros que hay que estimar; siendo $\alpha$ el parámetro de interés que nos da la estimación del impacto de la enseñanza del Análisis Crítico del Discurso.

Finalmente, la ecuación de la técnica de Diferencia en Diferencias queda así:

$$
E\left(Y_{\text {después }}-Y_{\text {antes }} \mid \text { tratamiento }\right)-E\left(Y_{\text {después }}-Y_{\text {antes }} \mid \text { control }\right)=\beta_{2}+\alpha-\beta_{2}=\alpha
$$




\section{RESULTADOS}

6.1. Nivel de comprensión de las ideologías en los estudiantes del segundo semestre de Sociología de la UNA-Puno, 2018 II, en la preprueba, en el grupo control y experimental.

Tabla 2 Resultados de preprueba del grupo control

\begin{tabular}{lcccc}
\hline \multicolumn{1}{c}{ Escala } & Intervalo & fi & Fi & \% \\
\hline Logro solvente & {$[20-18]$} & 0 & 0 & 0.0 \\
Logro previsto & {$[17-14]$} & 2 & 2 & 7.4 \\
En proceso & {$[13-11]$} & 10 & 12 & 37.0 \\
Evidencia dificultades & {$[10-0]$} & 15 & 27 & 55.6 \\
\hline Total & & 27 & & 100 \\
\hline
\end{tabular}

FUENTE: Elaboración propia con datos de la preprueba del grupo control

En la Tabla 2 se muestra el puntaje alcanzado por los estudiantes del grupo control en la preprueba (prueba de entrada). Como se puede observar, el 55.6\% de estudiantes obtuvo una nota entre 0 y 10 puntos. El 37\% de estudiantes obtuvo una nota entre 11 y 13 puntos. El 7.4\% de estudiantes obtuvo una nota entre 14 y 17 puntos.

Tabla 3 Resultados de preprueba del grupo experimental

\begin{tabular}{lcccc}
\hline \multicolumn{1}{c}{ Escala } & Intervalo & fi & Fi & \% \\
\hline Logro solvente & {$[20-18]$} & 0 & 0 & 0.0 \\
Logro previsto & {$[17-14]$} & 2 & 2 & 8.7 \\
En proceso & {$[13-11]$} & 10 & 12 & 43.5 \\
Evidencia dificultades & {$[10-0]$} & 11 & 23 & 47.8 \\
\hline Total & & 23 & & 100 \\
\hline
\end{tabular}

FUENTE: Elaboración propia con datos de la preprueba del grupo experimental

En la Tabla 3 se presentan los puntajes alcanzados por los estudiantes del grupo experimental en la preprueba (prueba de entrada). Se observa que el $47.8 \%$ de estudiantes obtuvo la nota entre 0 y 10 puntos. El $43.5 \%$ de estudiantes obtuvo la nota entre 11 y 13 puntos. El 8.7\% de estudiantes alcanzó la nota entre 14 y 17 puntos.

6.2 Nivel de comprensión de las ideologías en los estudiantes del segundo semestre de Sociología de la UNA-Puno, 2018 - II, en la posprueba, en el grupo control y experimental.

Tabla 4

Resultados de posprueba del grupo control

\begin{tabular}{|c|c|c|c|c|}
\hline Escala & Intervalo & $\mathbf{F i}$ & Fi & $\%$ \\
\hline Logro solvente & {$[20-18]$} & 0 & 0 & 0.0 \\
\hline Logro previsto & {$[17-14]$} & 3 & 3 & 11.1 \\
\hline En proceso & {$[13-11]$} & 11 & 14 & 40.7 \\
\hline Evidencia dificultades & {$[10-0]$} & 13 & 27 & 48.1 \\
\hline
\end{tabular}


FUENTE: Elaboración propia con datos de la posprueba del grupo control

En la Tabla 4 se muestran los puntajes alcanzados por los estudiantes del grupo control en la posprueba (prueba de salida). Se observa que el $48.1 \%$ de estudiantes obtuvo la nota entre 0 y 10 puntos. El $40.7 \%$ de estudiantes obtuvo la nota entre 11 y 13 puntos. El $11.1 \%$ de estudiantes alcanzó la nota entre 14 y 17 puntos.

Tabla 5 Resultados de posprueba del grupo experimental

\begin{tabular}{lcccc}
\hline \multicolumn{1}{c}{ Escala } & Intervalo & Fi & Fi & \% \\
\hline Logro solvente & {$[20-18]$} & 8 & 8 & 34.8 \\
Logro previsto & {$[17-14]$} & 12 & 20 & 52.2 \\
En proceso & {$[13-11]$} & 3 & 23 & 13.0 \\
Evidencia dificultades & {$[10-0]$} & 0 & 23 & 0.0 \\
\hline Total & & 23 & & 100 \\
\hline
\end{tabular}

FUENTE: Elaboración propia con datos de la posprueba del grupo experimental

En la Tabla 5 se presentan los puntajes alcanzados por los estudiantes del grupo experimental en la posprueba (prueba de salida). Como se puede avizorar, el 52.2\% de estudiantes obtuvo la nota entre 14 y 17 puntos. El 34.8\% de estudiantes alcanzó la nota entre 20 y 18 puntos. El 13\% de estudiantes obtuvo una nota entre 11 y 13 puntos.

\subsection{Resultados de la estimación de Diferencia en Diferencias}

Tabla 6 Resultados de la estimación de Diferencia en diferencias

\begin{tabular}{lccc} 
& Coeficiente & Prueba t & P> |t $\mid$ \\
\hline Tratamiento & -0.1771337 & -0.29 & 0.769 \\
Periodo & 0.4814815 & 0.83 & 0.407 \\
Interacción (impacto) & 5.127214 & 6.02 & 0.000 \\
cons & 10.48148 & 25.66 & 0.000 \\
\hline
\end{tabular}

$\begin{array}{rc}\mathrm{F}(3,96) & 37.47 \\ \text { Prob }>\mathrm{F} & 0.0000 \\ \text { R-squared } & 0.5394\end{array}$

Fuente: Elaboración propia sobre la base de los resultados calculados con el software Stata

En la Tabla 6 se presentan los resultados del modelo estadístico Diferencias en diferencias, que se ha estimado para determinar el impacto de la enseñanza del Análisis Crítico del Discurso como metodología de comprensión de las ideologías en los estudiantes de la Escuela Profesional de Sociología de la UNA - Puno, en el 2018 - II. Como se puede observar, el coeficiente de la variable interacción es 5.127214. Dicho valor es el impacto estimado. Lo cual significa que los estudiantes del grupo experimental tienen 5 puntos más que los estudiantes del grupo control. En cuanto a la prueba de significancia global se debe indicar que el valor de la prueba $F$ calculado (68.29) es mayor que el valor de la prueba F crítico, a un nivel de significancia de 1\%; por lo tanto, se rechaza la hipótesis nula y se acepta la hipótesis alterna. Eso quiere decir que el modelo estimado es significativo en términos estadísticos. En cuanto a la prueba de significancia individual, se debe señalar que el coeficiente de la variable interacción es significativo, ya que el valor de la prueba $t$ calculado (6.02) es mayor que el valor de la prueba $t$ crítico, a un nivel de significancia de $1 \%$. Lo cual quiere decir que el impacto es altamente significativo en términos estadísticos. 


\section{DISCUSIÓN}

Los resultados de la Tabla 2 y 3 sugieren que más de la mitad de los estudiantes se ubican en la escala Evidencia dificultades. Lo cual es alarmante, porque dichos estudiantes no poseen competencia de lectura crítica, es decir, no detectan la ideología del texto que leen. Por otro lado, un porcentaje mínimo de estudiantes se ubica en la escala Logro previsto. Lo cual guarda relación con el estudio de Díaz et al. (2015), quienes advirtieron ciertas habilidades de lectura crítica, la identificación de las intenciones del autor y el reconocimiento de fuentes de autoridad. Asimismo, los estudiantes (Tabla 2 y 3) no determinan la posición que el autor opta frente a su discurso; tampoco identifican los puntos de vista de los discursos, no logran situar el texto en el contexto sociocultural ni identifican los efectos del discurso en los lectores. Para enmendar dichos problemas se debería desarrollar tópicos referidos a la teoría de acto de habla, teorías de lectura crítica, pragmática, teoría de la enunciación y estrategias relacionadas con el análisis crítico del discurso y literacidad crítica, tal como señala Maya (2011) en su investigación. En consecuencia, los estudiantes en referencia requieren asistencia pedagógica (es decir, implementación de talleres de comprensión crítica de textos). Al respecto, Abouchaar (1997) subraya que la actividad pedagógica de enfoque crítico debe centrarse en orientar al lector a examinar los contenidos de lectura desde una perspectiva de diálogo abierto.

Los resultados de esta investigación coinciden con las conclusiones de Ochoa y Moreno (2013), quienes observan en su investigación que los estudiantes presentaron dificultades al asumir una posición crítica frente al contenido y forma del texto. Como señala Peña (2011), los problemas descritos en el párrafo anterior se presentan porque los estudiantes que ingresan a la universidad no tienen conocimientos previos requeridos para la lectura crítica. Otra causa posible sería porque la mayoría de los docentes concibe la lectura crítica de modo parcial y desenfocado, ya que se concentran solo en prácticas interpretativas y argumentativas, y no en procesos reflexivos, evaluativos y críticos (Avendaño de Barón, 2016). Al respecto, Castro y Martínez (2017) subrayan que algunos docentes reconocen la importancia de la lectura crítica, pero aún presentan ciertas dificultades para potenciar este nivel de comprensión lectora. Finalmente, Soria (2015) agrega que el 74\% de docentes no aplican motivación a su clase de lectura, ya que utilizan la metodología tradicionalista.

Los resultados de la Tabla 4 sugieren que casi la mitad de los estudiantes del grupo control siguen ubicándose en la escala Evidencia dificultades. Eso significa que no mejoraron en cuanto a la comprensión crítica de textos. Siguen presentando las mismas deficiencias, tanto en el nivel inferencial y crítico. Por lo tanto, se puede decir que estos estudiantes no poseen competencia adecuada para detectar las ideologías de los discursos. No conocen estrategias ni técnicas que les puede ayudar a leer apropiadamente los textos. Por lo tanto, es indispensable implementar la enseñanza de módulos sobre la literacidad crítica y análisis crítico del discurso sugeridos en esta investigación.

Los resultados de la Tabla 5 indican que más de la mitad de los estudiantes se ubican en la escala Logro previsto (con puntajes entre 14 y 17). Del mismo modo, más de la cuarta parte de estudiantes (con puntajes entre 18 y 20) se ubica en la escala Logro solvente. Eso significa que los estudiantes del grupo experimental mejoraron en cuanto a la comprensión crítica de textos, ya que ahora poseen competencias lectoras aceptables para leer las ideologías de los discursos. Como 
concluye Alvarado (2012), ahora los estudiantes reconocen que detrás del mensaje percibido existen determinadas posturas ideológicas. A estos estudiantes ya se les puede catalogar como lectores críticos, preparados para esta sociedad postmoderna y plural, donde la deconstrucción y análisis cultural e ideológico del discurso escrito son esenciales para el ejercicio de la ciudadanía (Serrano de Moreno y Madrid de Forero, 2007). Esta mejora se debe a que este grupo participó en el desarrollo de los ocho módulos de literacidad crítica y análisis crítico del discurso. Este resultado guarda relación con el estudio de Zárate (2019), quien concluye que la literacidad crítica ayuda a cuestionar el texto en vez de asumirlo pasivamente; a desarrollar interpretaciones alternativas y contextualizadas, y a diferenciar la opinión personal de la del autor. En consecuencia, los módulos desarrollados tuvieron un efecto positivo. Lo cual se demostró estadísticamente en la Tabla 6, donde se observa que el valor del impacto estimado es 5.127214. Lo cual quiere decir que los estudiantes del grupo experimental tienen 5 puntos más que los estudiantes del grupo control. De modo que la enseñanza de análisis crítico del discurso y la literacidad crítica tuvo un impacto positivo de 5 puntos en los estudiantes que formaron parte del grupo experimental.

\section{CONCLUSIÓN}

Según los resultados obtenidos, la enseñanza del análisis crítico del discurso tiene un impacto positivo en la comprensión de las ideologías en los estudiantes de segundo semestre de Sociología de la UNA - Puno, 2018 - II. Los estudiantes del grupo experimental mejoraron el nivel de comprensión de las ideologías en un promedio de 5 puntos a diferencia de los estudiantes del grupo control. Dichos datos se sustentan en evidencias estadísticas, ya que el impacto estimado según la técnica Diferencia en Diferencias fue de 5.127214 puntos. El cual es significativo en términos estadísticos a un nivel de $99 \%$ de confianza, porque el $P$-valor calculado resultó menor a 0.01. En consecuencias, el análisis crítico del discurso y la literacidad crítica es una herramienta de gran ayuda para comprender adecuadamente las ideologías de los discursos.

\section{REFERENCIAS BIBLIOGRÁFICAS}

Abouchaar, A. (1997). Lectura crítica: o cómo defenderse de la dictadura de la información. Ánfora, 5(10), 18-28.

Aguilar, L. (2008). Lecturas transversales para formar receptores críticos. Comunicar, XVI(31), 2733. https://doi.org/10.3916/c31-2008-01-003

Alvarado, M. (2012). Lectura crítica de medios: una propuesta metodológica. Comunicar, XX(39), 101-108. https://doi.org/10.3916/C39-2012-02-10

Arias, G. (2018). La lectura crítica como estrategia para el desarrollo del pensamiento lógico. Revista Boletín Redipe, 7(1), 86-94.

Avendaño de Barón, G. (2016). La lectura crítica en Educación Básica Secundaria y Media: la voz de los docentes. Cuadernos de Lingüística Hispánica, (28), 207-232. Retrieved from http://www.redalyc.org/articulo.oa?id=322246463011

Barrio, M., \& Pérez, P. (1997). Desmitificar la publicidad: lectura crítica de los medios en el aula. Comunicar, (9), 130-133. Retrieved from http://www.redalyc.org/articulo.oa?id=15800919

Benavides, D., \& Sierra, G. (2013). Estrategias didácticas para fomentar la lectura crítica desde la 
perspectiva de la transversalidad. REICE. Revista Iberoamericana Sobre Calidad, Eficacia y Cambio En Educación E-ISSN:, 11(3), 79-109.

Calsamiglia, H., \& Tusón, A. (1999). Las cosas del decir. Manual de análisis del discurso. Barcelona: Editorial Ariel, S. A.

Cardona, P., \& Londoño, D. (2017). El sentido de la lectura crítica en contexto. Revista Katharsis, (22), 375-401. Retrieved from http://revistas.iue.edu.co/index.php/katharsis/index

Cassany, D. (2005). Literacidad crítica: leer y escribir la ideología. II Congreso Internacional y IV Foro Nacional de Enseñanza de La Lengua y La Literatura, 89-96. Retrieved from https://www.researchgate.net/publication/251839730_Literacidad_critica_leer_y_escribir_la_id eologia

Cassany, D. (2006). Tras las Lineas. Sobre la lectura contemporanea. Barcelona: Anagrama.

Castro, J., \& Martínez, M. (2017). La lectura crítica en ELE: una mirada desde el docente y sus creencias. Pontificia Universidad Javeriana.

Chillón, A. (1998). El giro linguístico y su incidencia en el estudio de la comunicación peridística. Anàlisi: Quaderns de Comunicació $i$ Cultura, (22), 63-98. Retrieved from https://www.raco.cat/index.php/analisi/article/viewFile/14987/14828

Díaz, J., Bar, A., \& Ortiz, M. (2015). La lectura crítica y su relación con la formación disciplinar de estudiantes universitarios. Revista de La Educación Superior, 44(176), 139-158. https://doi.org/10.1016/j.resu.2015.12.006

Fainholc, B. (2006). La lectura crítica en Internet : evaluación y aplicación de sus recursos.

García, C. (2014). Introducción a la lectura crítica de artículos : dise no de estudios y sesgos. Radiología. https://doi.org/10.1016/j.rx.2014.08.002

Guzmán, F. (2016). Medios de comunicación y representación de conflictos étnico-sociales en Perú. Universitat Pompeu Fabra.

Martínez, E. (1995). Educación para la lectura crítica de la televisión. Comunicar, (4), 42-51.

Maya, L. (2011). Una práctica de lectura crítica con los estudiantes de la Universidad de Medellín. Revista Virtual Universidad Católica Del Norte, (34), 162-193. Retrieved from http://www.redalyc.org/articulo.oa?id=194222473008

Méndez, J., Arbeláez, D., Serna, C., Espinal, C., \& Gómez, J. (2014). La lectura crítica en la educación superior: un estado de la cuestión. Revista Virtual Universidad Católica Del Norte, (41), 4-18. Retrieved from http://revistavirtual.ucn.edu.co/index.php/RevistaUCN/article/view/461/983

Ochoa, L., \& Moreno, E. (2013). Lectura crítica en estudiantes universitarios. Revista PAPELES, 5(9), $31-44$.

Peña, F. (2011). Leer y escribir. Prácticas necesarias en la universidad. Educere, 15(52), 711-719. Retrieved from http://www.redalyc.org/articulo.oa?id=35622379018

Rocha, Y. (2016). La lectura crítica: encuentro de subjetividades (Universidad de Antioquía). Retrieved from url

Romano, M., \& López, E. (2016). Lectura crítica de medios en estudiantes universitarios. Praxis Educativa, 20(2), 25-33. https://doi.org/10.19137/praxiseducativa-2016-200203 Lectura

Serrano de Moreno, S. (2008). El desarrollo de la comprensión crítica en los estudiantes universitarios: Hacia una propuesta didáctica. Educere, 12(42), 505-514.

Serrano de Moreno, S., \& Madrid de Forero, A. (2007). Competencias de lectura crítica. Una propuesta para la refl exión y la práctica Serrano. Acción Pedagógica, 16(1), 58-68.

Silva, C. (2002). Lecturas críticas de un mensaje cinematográfico. Comunicar, (19), 92-96. Retrieved 
from http://www.redalyc.org/articulo.oa?id=15801917

Soria, D. E. (2015). Diseño de Estrategias Metodológicas para mejorar la Lectura Crítica para el área de Lengua y Literatura en cuarto nivel de Educación Básica. Pontificia Universidad Católica del Ecuador.

Van Dijk, T. (1994). Discurso, Poder y Cognición Social. Cuadernos de La Maestría En Lingüística. Retrieved from http://www.discursos.org/oldarticles/Discurso, poder y cognici\%F3n social.pdf

Van Dijk, T. (1999). El análisis crítico del discurso. Anthropos, (186), 23-36.

Van Dijk, T. (2003). Racismo y discurso de las élites. Barcelona: Gedisa.

Van Dijk, T. (2006). Ideología. Una aproximación multidisciplinaria. Barcelona: Gedisa.

Van Dijk, T. (2008a). El estudio del discurso. In El discurso como estructura y proceso. Barcelona: Gedisa.

Van Dijk, T. (2008b). Semántica del discurso e ideología. Discurso y Sociedad, 1(2), 201-261.

Van Dijk, T. (2009). Discurso y poder. Barcelona: Gedisa.

Van Dijk, T. (2016). Discurso-cognição-sociedade: estado atual e perspectivas da abordagem sociocognitiva do discurso. Letronica, (9), 8-29. https://doi.org/10.15448/19844301.2016.s.23189

Vich, V., \& Zavala, V. (2004). Oralidad y poder. Herramientas metodologicas. Bogotá: Grupo Editorial Norma.

Wodak, R. (1987). And Where Is the Lebanon? A Socio-Psycholinguistic Investigation of Comprehension and intelligibility of News. Text, 7(4), 377-410.

Wodak, R. (2003). De qué trata el análisis crítico del discurso (ACD). Resumen de su historia, sus conceptos fundamentales y sus desarrollos. In R. Wodak \& M. Meyer (Eds.), Métodos de análisis crítico del discurso. Barcelona: Gedisa.

Zárate, A. (2019). Habilidades de lectura crítica en los libros de texto de educación secundaria. Revista Signos, 52(99), 181-206. https://doi.org/10.4067/S0718-09342019000100181 
Apéndice 1

Módulos de la enseñanza y aplicación de análisis crítico del discurso y literacidad crítica

\begin{tabular}{|c|c|c|c|c|}
\hline \multirow[t]{2}{*}{ MÓDULOS } & \multirow[t]{2}{*}{ CAMPO TEMÁTICO } & \multicolumn{3}{|c|}{ TIEMPO } \\
\hline & & Teoría & Práctica & Total horas \\
\hline $\begin{array}{l}\text { MODULO I } \\
\text { Pragmática del } \\
\text { discurso }\end{array}$ & $\begin{array}{l}\text { Actos de habla } \\
\text { Implicaturas } \\
\text { Contexto }\end{array}$ & 3 & 1 & 4 \\
\hline $\begin{array}{l}\text { MODULO II } \\
\text { Las personas del } \\
\text { discurso }\end{array}$ & $\begin{array}{l}\text { Enunciación } \\
\text { Deixis: marcadores de la enunciación } \\
\text { Polifonía de Ducrot }\end{array}$ & 3 & 1 & 4 \\
\hline $\begin{array}{l}\text { MODULO III } \\
\text { Análisis crítico del } \\
\text { discurso }\end{array}$ & $\begin{array}{l}\text { Noción de discurso en ACD } \\
\text { Dimensiones de ACD } \\
\text { Tópicos de ACD según van Dijk } \\
\text { Ideología }\end{array}$ & 3 & 1 & 4 \\
\hline $\begin{array}{l}\text { MODULO IV } \\
\text { Literacidad crítica }\end{array}$ & $\begin{array}{l}\text { Nociones de literacidad crítica } \\
22 \text { técnicas de Cassany }\end{array}$ & 3 & 1 & 4 \\
\hline $\begin{array}{l}\text { MODULO V } \\
\text { Guía de literacidad } \\
\text { crítica }\end{array}$ & $\begin{array}{l}\text { Explicación teórica y práctica de la guía } \\
\text { de literacidad crítica }\end{array}$ & 1 & 3 & 4 \\
\hline $\begin{array}{l}\text { MODULO VI } \\
\text { Interpretación del } \\
\text { texto } 1\end{array}$ & $\begin{array}{l}\text { Lectura de texto } 1 \\
\text { Aplicación de la guía de literacidad } \\
\text { crítica }\end{array}$ & 0 & 2 & 2 \\
\hline $\begin{array}{l}\text { MODULO VII } \\
\text { Interpretación del } \\
\text { texto } 2\end{array}$ & $\begin{array}{l}\text { Lectura de texto } 2 \\
\text { Aplicación de la guía de literacidad } \\
\text { crítica }\end{array}$ & 0 & 2 & 2 \\
\hline $\begin{array}{l}\text { MODULO VIII } \\
\text { Interpretación del } \\
\text { texto } 3\end{array}$ & $\begin{array}{l}\text { Lectura de texto } 3 \\
\text { Aplicación de la guía de literacidad } \\
\text { crítica }\end{array}$ & 0 & 2 & 2 \\
\hline TOTAL & & 13 & 13 & 26 \\
\hline
\end{tabular}

Nota: Elaboración propia 


\section{Apéndice 2}

\section{Rubrica de evaluación de nivel de comprensión de las ideologías}

\begin{tabular}{|c|c|c|c|c|c|}
\hline Dimensiones & Indicadores & $\begin{array}{l}\text { Excelente } \\
\text { (2) }\end{array}$ & $\begin{array}{c}\text { Bueno } \\
(1.5)\end{array}$ & $\begin{array}{c}\text { Regular } \\
\text { (1) }\end{array}$ & $\begin{array}{l}\text { Deficiente } \\
\text { (0) }\end{array}$ \\
\hline \multirow{3}{*}{ 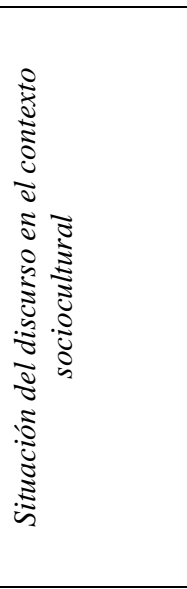 } & $\begin{array}{l}\text { Identificación } \\
\text { del propósito } \\
\text { del autor }\end{array}$ & $\begin{array}{l}\text { Identifica } \\
\text { plenamente el } \\
\text { propósito del } \\
\text { autor }\end{array}$ & $\begin{array}{l}\text { Identifica } \\
\text { razonablemente el } \\
\text { propósito del } \\
\text { autor }\end{array}$ & $\begin{array}{l}\text { Identifica } \\
\text { parcialmente el } \\
\text { propósito del autor }\end{array}$ & $\begin{array}{l}\text { No identifica el } \\
\text { propósito del autor }\end{array}$ \\
\hline & $\begin{array}{l}\text { Detección del } \\
\text { posicionamiento }\end{array}$ & $\begin{array}{l}\text { Detecta } \\
\text { plenamente el } \\
\text { posicionamiento } \\
\text { del autor frente a } \\
\text { las } \\
\text { representaciones } \\
\text { culturales }\end{array}$ & $\begin{array}{l}\text { Detecta } \\
\text { razonablemente el } \\
\text { posicionamiento } \\
\text { del autor frente a } \\
\text { las } \\
\text { representaciones } \\
\text { culturales }\end{array}$ & $\begin{array}{l}\text { Detecta } \\
\text { parcialmente el } \\
\text { posicionamiento } \\
\text { del autor frente a } \\
\text { las } \\
\text { representaciones } \\
\text { culturales }\end{array}$ & $\begin{array}{l}\text { No detecta el } \\
\text { posicionamiento del } \\
\text { autor frente a las } \\
\text { representaciones } \\
\text { culturales }\end{array}$ \\
\hline & $\begin{array}{l}\text { Mapa } \\
\text { sociocultural }\end{array}$ & $\begin{array}{l}\text { Identifica } \\
\text { plenamente lo que } \\
\text { el texto oculta } \\
\text { sobre el tema que } \\
\text { trata }\end{array}$ & $\begin{array}{l}\text { Identifica } \\
\text { razonablemente } \\
\text { lo que el texto } \\
\text { oculta }\end{array}$ & $\begin{array}{l}\text { Identifica } \\
\text { parcialmente lo que } \\
\text { el texto oculta }\end{array}$ & $\begin{array}{l}\text { No identifica lo que el } \\
\text { texto oculta sobre el } \\
\text { tema que trata }\end{array}$ \\
\hline \multirow{4}{*}{ 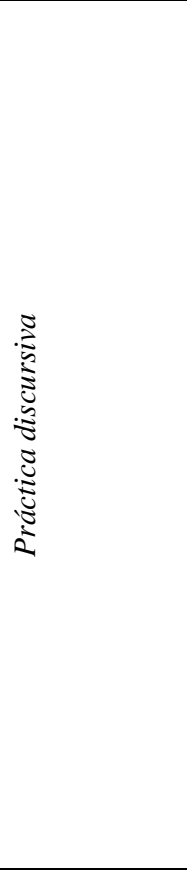 } & $\begin{array}{l}\text { Identificación } \\
\text { del género } \\
\text { discursivo }\end{array}$ & $\begin{array}{l}\text { Identifica } \\
\text { plenamente el } \\
\text { género del } \\
\text { discurso e } \\
\text { interpreta el } \\
\text { discurso según } \\
\text { dicho género. } \\
\end{array}$ & $\begin{array}{l}\text { Identifica el } \\
\text { género del } \\
\text { discurso, pero no } \\
\text { interpreta el } \\
\text { discurso según } \\
\text { dicho género. }\end{array}$ & $\begin{array}{l}\text { Identifica } \\
\text { parcialmente el } \\
\text { género del discurso }\end{array}$ & $\begin{array}{l}\text { No identifica el género } \\
\text { del discurso }\end{array}$ \\
\hline & $\begin{array}{l}\text { Identificación } \\
\text { de contrincantes }\end{array}$ & $\begin{array}{l}\text { Identifica } \\
\text { plenamente los } \\
\text { contrincantes } \\
\text { contra quienes } \\
\text { escribe el autor }\end{array}$ & $\begin{array}{l}\text { Identifica } \\
\text { razonablemente } \\
\text { los contrincantes } \\
\text { contra quienes } \\
\text { escribe el autor }\end{array}$ & $\begin{array}{l}\text { Identifica } \\
\text { parcialmente los } \\
\text { contrincantes } \\
\text { contra quienes } \\
\text { escribe el autor } \\
\end{array}$ & $\begin{array}{l}\text { No identifica los } \\
\text { contrincantes contra } \\
\text { quienes escribe el autor }\end{array}$ \\
\hline & $\begin{array}{l}\text { Solidez y fuerza } \\
\text { del discurso }\end{array}$ & $\begin{array}{l}\text { Determina el tipo } \\
\text { de razonamiento y } \\
\text { pertenencia de los } \\
\text { datos y ejemplos }\end{array}$ & $\begin{array}{l}\text { Determina el tipo } \\
\text { de razonamiento, } \\
\text { y, parcialmente, } \\
\text { la pertenencia de } \\
\text { los datos y } \\
\text { ejemplos }\end{array}$ & $\begin{array}{l}\text { Determina } \\
\text { parcialmente el tipo } \\
\text { de razonamiento, y } \\
\text { no determina la } \\
\text { pertenencia de } \\
\text { datos y ejemplos }\end{array}$ & $\begin{array}{l}\text { No determina el tipo de } \\
\text { razonamiento ni la } \\
\text { pertenencia de datos y } \\
\text { ejemplos }\end{array}$ \\
\hline & $\begin{array}{l}\text { Jerarquía } \\
\text { informativa }\end{array}$ & $\begin{array}{l}\text { Diferencia } \\
\text { plenamente la } \\
\text { información } \\
\text { destacada de la } \\
\text { información } \\
\text { secundaria }\end{array}$ & $\begin{array}{l}\text { Diferencia } \\
\text { razonablemente la } \\
\text { información } \\
\text { destacada de la } \\
\text { información } \\
\text { secundaria }\end{array}$ & $\begin{array}{l}\text { Diferencia } \\
\text { parcialmente la } \\
\text { información } \\
\text { destacada de la } \\
\text { información } \\
\text { secundaria }\end{array}$ & $\begin{array}{l}\text { No diferencia la } \\
\text { información destacada } \\
\text { de la información } \\
\text { secundaria }\end{array}$ \\
\hline \multirow{3}{*}{ 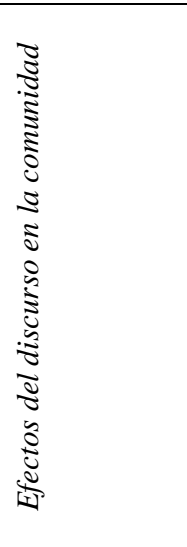 } & $\begin{array}{l}\text { La sombra del } \\
\text { lector }\end{array}$ & $\begin{array}{l}\text { Identifica } \\
\text { plenamente el tipo } \\
\text { de lector a quien } \\
\text { está dirigido el } \\
\text { texto }\end{array}$ & $\begin{array}{l}\text { Identifica } \\
\text { razonablemente el } \\
\text { tipo de lector a } \\
\text { quien está } \\
\text { dirigido el texto }\end{array}$ & $\begin{array}{l}\text { Identifica } \\
\text { parcialmente el tipo } \\
\text { de lector a quien } \\
\text { está dirigido el } \\
\text { texto }\end{array}$ & $\begin{array}{l}\text { No identifica el tipo de } \\
\text { lector a quien está } \\
\text { dirigido el texto }\end{array}$ \\
\hline & $\begin{array}{l}\text { Acuerdos y } \\
\text { desacuerdos }\end{array}$ & $\begin{array}{l}\text { Plantea con } \\
\text { mucha solvencia } \\
\text { académica sus } \\
\text { desacuerdos con } \\
\text { el autor }\end{array}$ & $\begin{array}{l}\text { Plantea con poca } \\
\text { solvencia } \\
\text { académica sus } \\
\text { desacuerdos con } \\
\text { el autor }\end{array}$ & $\begin{array}{l}\text { Intenta plantear sus } \\
\text { desacuerdos con el } \\
\text { autor }\end{array}$ & $\begin{array}{l}\text { No plantea ningún } \\
\text { desacuerdo con el autor }\end{array}$ \\
\hline & $\begin{array}{l}\text { Interpretaciones } \\
\text { de los otros }\end{array}$ & $\begin{array}{l}\text { Sugiere distintas } \\
\text { interpretaciones } \\
\text { posibles según } \\
\text { otros contextos }\end{array}$ & $\begin{array}{l}\text { Sugiere } \\
\text { aceptablemente } \\
\text { una interpretación } \\
\text { Posible según } \\
\text { otro contexto }\end{array}$ & $\begin{array}{l}\text { Sugiere } \\
\text { parcialmente otra } \\
\text { interpretación } \\
\text { posible }\end{array}$ & $\begin{array}{l}\text { No sugiere ninguna otra } \\
\text { interpretación posible } \\
\text { según otros contextos }\end{array}$ \\
\hline
\end{tabular}

Nota: Elaboración propia sobre la base de la propuesta de Cassany $(2005,2006)$. 


\section{Apéndice 3}

\section{Guía de aplicación de literacidad crítica}

\begin{tabular}{|l|l|}
\hline $\begin{array}{l}\text { 1. Situación del discurso en el contexto } \\
\text { sociocultural }\end{array}$ & Respuestas \\
\hline Propósito del autor \\
¿Qué quiere el autor? ¿qué desea cambiar? ¿qué & \\
espera del lector? & \\
\hline Retrato del autor & \\
¿Qué sabes del autor?, ¿cómo se le valora en tu & \\
comunidad?, ipor qué escribe? & \\
\hline Subjetividad del autor \\
¿Qué nos dice de sí mismo el autor? ¿Qué imagen & \\
proyecta de sí mismo en su discurso? & \\
\hline Posicionamiento \\
¿El autor es sexista?, ¿hay rasgos xenófobos?, ¿hay \\
rasgos etnocentristas (raza)?, ¿es respetuoso de todas \\
las identidades?, ¿qué palabras ha elegido para \\
enfrentarse a las controversias?, ¿cuáles son los \\
estereotipos culturales que maneja?, ¿qué aspectos \\
religiosos, políticos, educativos, sociales y culturales \\
presupone que todos tenemos o compartimos?
\end{tabular}

\begin{tabular}{|l|l|}
\hline 2. Práctica discursiva & Respuestas \\
\hline Género discursivo \\
¿Qué tipo de texto se está leyendo? (noticia, blog, & \\
artículo científico, ensayo, texto de opinión, carta & \\
personal...) ¿el autor utiliza los recursos & \\
convencionales o criterios del género? ¿sigue una & \\
tradición establecida? & \\
\hline Identificación de contrincantes \\
¿Contra quién escribe el autor?, ¿qué personas se & \\
oponen a sus deseos y pensamientos?, ¿Qué grupos & \\
o personas coinciden con el autor? & \\
\hline Voces (citas) & \\
¿Cuáles son las citas literales, indirectas o & \\
encubiertas? El autor reutiliza voces favorables; las & \\
que hablan en su contra, las que le critican, las hace & \\
callar, las olvida. & \\
\hline Solidez y fuerza del discurso \\
¿De qué tipo son los razonamientos?, es decir, ¿el & \\
autor apela a los sentimientos o a las & \\
argumentaciones?, ¿los ejemplos son pertinentes? & \\
¿Los datos son claros, actualizados, pertinentes y & \\
tienen una fuente identificable? & \\
\hline Jerarquía informativa \\
¿Cuáles son los datos destacados y cuáles se & \\
presentan como detalles?, ¿cuáles son los datos & \\
indiscutibles y los matizados?, ¿los centrales y los & \\
marginales? & \\
\hline
\end{tabular}




\begin{tabular}{|c|c|}
\hline 3. Efectos del discurso en la comunidad & Respuestas \\
\hline \multicolumn{2}{|l|}{$\begin{array}{l}\text { Propósitos del lector } \\
\text { ¿Qué buscas en el texto como lector? ¿Por qué lo } \\
\text { lees? ¿Qué esperas encontrar en él? Luego de leerlo, } \\
\text { ¿encontraste lo que buscabas? }\end{array}$} \\
\hline \multicolumn{2}{|l|}{$\begin{array}{l}\text { La sombra del lector } \\
\text { ¿A quién se dirige el texto?, ¿por qué se dirige a } \\
\text { este tipo de lector y no a otro?, ¿qué presupone que } \\
\text { el lector sabe o no sabe?, ¿qué datos expone (por } \\
\text { qué el autor ha decidido que el lector no sabe y debe } \\
\text { explicárselo)? ¿Cuál es el perfil del destinatario del } \\
\text { texto? ¿Qué imagen del lector proyecta el discurso? }\end{array}$} \\
\hline \multicolumn{2}{|l|}{$\begin{array}{l}\text { Acuerdos y desacuerdos } \\
\text { Relee el texto y compara lo que dice con lo que tú } \\
\text { crees. Marca con un signo de sumar }(+) \text { los puntos } \\
\text { en que estés de acuerdo, y con un signo de restar (-) } \\
\text { los puntos en que discrepes. ¿Cuántos + y cuántos - } \\
\text { hay?, ¿por qué? }\end{array}$} \\
\hline \multicolumn{2}{|l|}{$\begin{array}{l}\text { Interpretaciones de otros } \\
\text { ¿Cómo van a leer el texto otros lectores? Sugiere } \\
\text { distintas interpretaciones posibles según otros } \\
\text { contextos. }\end{array}$} \\
\hline \multicolumn{2}{|l|}{$\begin{array}{l}\text { Aspectos controvertidos } \\
\text { ¿Cuáles son las partes más controvertidas del texto? } \\
\text { ¿Qué efecto causa en su conjunto? ¿Cuál es la } \\
\text { impresión general? }\end{array}$} \\
\hline $\begin{array}{l}\text { Reacciones frente al texto } \\
\text { ¿Qué se va a hacer con el texto?, ¿se olvidará, se } \\
\text { replicará, se responderá? Valora cuál puede ser la } \\
\text { reacción más apropiada según tus intereses. }\end{array}$ & \\
\hline
\end{tabular}

Nota. Esta guía fue adaptada de las 22 técnicas de Cassany (2006). 